\title{
Increasing genomic diversity and evidence of constrained lifestyle evolution due to insertion sequences in Aeromonas salmonicida
}

\author{
Antony T. Vincent ${ }^{1,2,3}$, Mélanie V. Trudel ${ }^{1,2,3}$, Luca Freschi ${ }^{1,4}$, Vandan Nagar ${ }^{5}$, Cynthia Gagné-Thivierge ${ }^{1,2,3}$,
} Roger C. Levesque ${ }^{1,4}$ and Steve J. Charette ${ }^{1,2,3^{*}}$

\begin{abstract}
Background: Aeromonads make up a group of Gram-negative bacteria that includes human and fish pathogens. The Aeromonas salmonicida species has the peculiarity of including five known subspecies. However, few studies of the genomes of A. salmonicida subspecies have been reported to date.

Results: We sequenced the genomes of additional A. salmonicida isolates, including three from India, using nextgeneration sequencing in order to gain a better understanding of the genomic and phylogenetic links between $A$. salmonicida subspecies. Their relative phylogenetic positions were confirmed by a core genome phylogeny based on 1645 gene sequences. The Indian isolates, which formed a sub-group together with A. salmonicida subsp. pectinolytica, were able to grow at either at $18{ }^{\circ} \mathrm{C}$ and $37^{\circ} \mathrm{C}$, unlike the $A$. salmonicida psychrophilic isolates that did not grow at $37^{\circ} \mathrm{C}$. Amino acid frequencies, GC content, tRNA composition, loss and gain of genes during evolution, pseudogenes as well as genes under positive selection and the mobilome were studied to explain this intraspecies dichotomy.

Conclusion: Insertion sequences appeared to be an important driving force that locked the psychrophilic strains into their particular lifestyle in order to conserve their genomic integrity. This observation, based on comparative genomics, is in agreement with previous results showing that insertion sequence mobility induced by heat in $A$. salmonicida subspecies causes genomic plasticity, resulting in a deleterious effect on the virulence of the bacterium. We provide a proof-of-concept that selfish DNAs play a major role in the evolution of bacterial species by modeling genomes.
\end{abstract}

Keywords: Aeromonas salmonicida, Phylogeny, Mesophilic, Psychrophilic, Insertion sequence

\section{Background}

The Aeromonas genus (also known as aeromonads) has a complex taxonomy, with fourteen species officially recognized in the latest edition of the Bergey's Manual of Systematic Bacteriology [1]. However, recent works like

\footnotetext{
*Correspondence: steve.charette@bcm.ulaval.ca

'Institut de biologie intégrative et des systèmes, Pavillon

Charles-Eugène-Marchand, Université Laval, 1030 avenue de la Médecine,

Quebec City G1V OA6QC, Canada

${ }^{2}$ Centre de recherche de l'Institut universitaire de cardiologie et de pneumologie de Québec (Hôpital Laval), 2725 Chemin Sainte-Foy, Quebec City G1V 4G5QC, Canada

Full list of author information is available at the end of the article
}

the one of Colston et al. in 2014 showed, based on molecular phylogenies (house-keeping genes and core genomes), average nucleotide identities (ANI), and in silico DNA-DNA hybridization (isDDH), that aeromonads have a greater taxonomic richness [2].

Aeromonads can be also divided into two groups based on their ability or inability to grow at a mesophilic temperature $\left(37^{\circ} \mathrm{C}\right)[3-5]$. The best-known representatives of the mesophilic group are A. hydrophila, $A$. caviae, and $A$. veronii, all of which are human pathogens $[3,4]$. The psychrophilic group (constituted by species that cannot grow at $37{ }^{\circ} \mathrm{C}$ ) includes A. salmonicida, 
which can be further divided into five subspecies: salmonicida, smithia, achromogenes, and masoucida [3] as well as pectinolytica, which is an exception to the rule since it grows well at $37{ }^{\circ} \mathrm{C}$ and is considered to be mesophilic [6].

A. salmonicida is of particular interest since this aeromonad species is known to include fish pathogens causing important economical loss worldwide [7, 8]. The genomes of all the subspecies have been sequenced and have been deposited in public databases such as GenBank or Sequence Read Archive (SRA). The sequenced isolates come from geographical origins as diverse as Argentina, Chile, Canada, the United Kingdom, France, Switzerland, Korea, and Japan. However, among these isolates only the genome of $A$. salmonicida subsp. salmonicida A449 was completely sequenced, assembled and annotated [9]. A striking result of this study was the discovery of 10 types of insertion sequences (ISs), which were present in 102 copies (88 complete and 14 partial), compared to the genome of $A$. hydrophila, which contained no ISs [10].

Recently, A. salmonicida isolates have also been obtained in India from food samples at a local market [11]. The origin of these A. salmonicida isolates (i.e., not directly from water or diseased fish) in a tropical climate is very intriguing in terms of the spread of A. salmonicida species. With the exception of the pectinolytica subsp., which was isolated from a polluted river in Argentina [6], all the other $A$. salmonicida subsp. were isolated from infected hosts living in cold water [9, 12-17].

We sequenced the genomes and examined the genomic elements of the Indian isolates in order to gain a better understanding of the genome architecture and the wide diversity of $A$. salmonicida species. Consequently, we performed an optimized core-genome phylogeny of the aeromonads including previously sequenced $A$. salmonicida genomes as well as genomes sequenced specifically for the present study. A comparison of these genomes revealed that among aeromonads, A. salmonicida subspecies are unusually diverse. It also uncovered at least one new mesophilic subspecies and showed that A. salmonicida was evolving from a mesophilic to a psychrophilic lifestyle due to the loss of its ability to proliferate in mesophilic environments. We propose that insertion sequences are an important driving force, which may have locked some $A$. salmonicida subspecies into a psychrophilic lifestyle in order to conserve their genomic integrity.

\section{Results and discussion}

\section{Sequencing new aeromonad genomes}

To shed light on the evolution and diversity of $A$. salmonicida species, we first used next-generation sequencing (NGS) to sequence the complete genomes of five additional isolates, including one member of the subspecies smithia (JF4097), one of the subspecies salmonicida (RS 534), and three isolates from India with an unclear taxonomy (Y47, Y567, and Y577) [11]. The results of the de novo assembly for the five isolates are presented in Additional file 1.

\section{Phylogenetic analysis of $A$. salmonicida}

A robust optimized molecular phylogeny of 43 aeromonads was inferred from 1645 gene sequences (see Additional files 1 and 2) to study the taxonomy of this genus and more specifically the salmonicida species. Since our study mainly focused on $A$. salmonicida, we were surprised to see that according to our phylogeny, A. salmonicida CBA100, a recently deposited Chilean isolate [12], was phylogenetically closer to A. bestiarum than to A. salmonicida (see Additional file 1). To verify the relatedness of the CBA100 isolate and $A$. bestiarum, the average nucleotide identity (ANI) values were computed for some key taxa (see Additional file 1). The ANI values were in agreement with the molecular phylogeny since the ANI value of the CBA100 isolate and $A$. bestiarum was above $96 \%$, meaning that the CBA100 isolate likely belong to the $A$. bestiarum species and not $A$. salmonicida as initially proposed [12]. A recent study based on in silico DNA-DNA hybridization (isDDH) also suggested that CBA100 isolate is a member of the A. bestiarum group [18].

The Indian isolates (Y47, Y567, and Y577) had basal positions among the salmonicida species (Fig. 1) and the ANI analysis confirmed that these taxa are salmonicida species (see Additional file 1). Isolate Y577 shared a clade with A. salmonicida subsp. pectinolytica while isolates Y47 and Y567 formed a basal clade to the masoucida subspecies. The subspecies smithia formed a clade with the subspecies achromogenes while the subspecies salmonicida RS 534 strain clustered among the other isolates of this subspecies (Fig. 1).

The complete taxon sampling of the salmonicida subspecies coupled with the high level of accuracy engendered by the high number of markers $(696,249$ positions and 420,006 alignment patterns) of our molecular phylogenetic analysis clustered the European and the Canadian isolates independently (Fig. 1). It is interesting to note that the bootstrap value at the node corresponding to the split between the European and the Canadian isolates is 100, which suggests an important statistical robustness. Previous studies using low resolution approaches such as restriction fragment length polymorphism (RFLP) and random amplified polymorphic DNA (RAPD) have indicated that salmonicida subspecies strains are genetically homogeneous [19-22] and with a clonal population structure $[23,24]$. Another study, based on the sequence of the gene $\operatorname{tap} A$, proposed that there was no difference between 


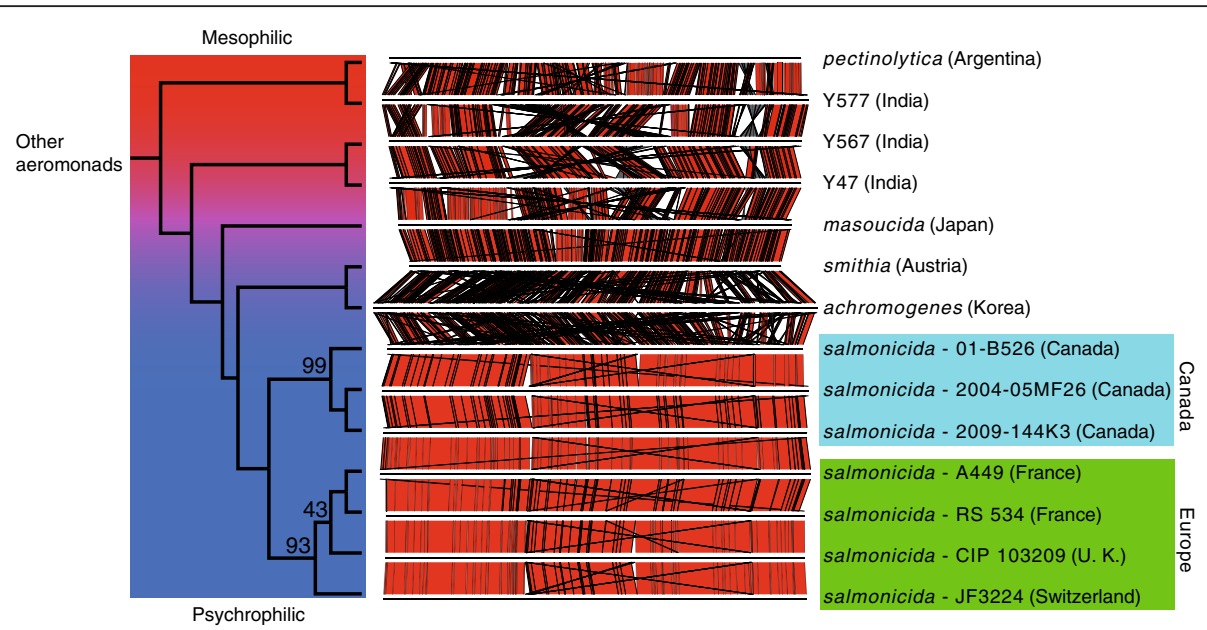

Fig. 1 View of the salmonicida species section of the phylogenetic tree with proportional branch lengths. See Additional file 1 for the complete tree for all the aeromonads. Putative chromosome sequences were constructed for all the taxa and were compared based on their phylogenetic positions. The red alignments show identity between direct sequences while the gray ones show between inverted sequences. Only the subspecies of each A. salmonicida strain are indicated on the right. The geographic provenance of each taxon is indicated in brackets. Only bootstrap values below 100 are shown. The gradient from red to blue represents the ability of each taxon to grow at a mesophilic temperature (red) or at a strict psychrophilic one (blue)

the European isolates and those found in North America [25]. However, recent studies have shown that isolates from different geographical regions may bear specific variants of the AsaGEI genomic island and that AsaGEI can be used to track the geographical provenance of salmonicida isolates $[15,26]$. The present study confirms that there are differences between European and Canadian isolates at the molecular level. On the other hand, the branch lengths were small (see Additional file 1), meaning that the dichotomy between the European and Canadian isolates is a relatively recent event and/or that the European and Canadian isolates have a similar mutation rate. Additional salmonicida isolates from other regions need to be analyzed to determine whether they also cluster differently.

\section{Mesophilic/psychrophilic dichotomy}

Since A. salmonicida subsp. pectinolytica is a mesophilic strain and is closely related to the Indian isolates (Fig. 1), we grew the Indian isolates (Y47, Y567 and Y577) at $18^{\circ} \mathrm{C}$ and $37^{\circ} \mathrm{C}$ to clarify whether they were mesophilic as well. They grew very well at $37^{\circ} \mathrm{C}$ and could thus be considered as mesophilic (Fig. 2a). A. salmonicida subsp. masoucida, which is considered a psychrophilic subspecies [3], tolerated and even grew at $37{ }^{\circ} \mathrm{C}$ albeit slowly and to a lower density before declining. This capacity of $A$. salmonicida subsp. masoucida to grow moderately at $37{ }^{\circ} \mathrm{C}$ was also reported in a previous large-scale phenotypic study without, however, being clearly mentioned [27]. This is a key observation given that $A$. salmonicida subsp. masoucida is positioned in the molecular phylogeny directly after the mesophilic clades and shares the same basal node as the psychrophilic clades (Fig. 1).

The $18{ }^{\circ} \mathrm{C}$ temperature has been chosen for additional growth tests since it is at this temperature that psychrophilic A. salmonicida subsp. salmonicida strains are usually the more efficient to infect fish [28-30]. All the tested isolates, including the mesophiles, grew well at $18{ }^{\circ} \mathrm{C}$ (Fig. 2b). Even if $18{ }^{\circ} \mathrm{C}$ is the most efficient temperature for A. salmonicida to infect fish, we have also tested the growth of these isolates at $7{ }^{\circ} \mathrm{C}$. The growth curves showed the same patterns than at $18{ }^{\circ} \mathrm{C}$, with the mesophilic strains growing more efficiently than the psychrophilic ones (see Additional file 1). Interestingly, the psychrophilic isolates had a lower growth rate capacity than the mesophilic ones at psychrophilic temperatures. This suggests that the psychrophilic isolates did not gain the ability to be psychrophilic but, in fact, experienced an alteration of their overall physiology leading to the loss of their capacity to grow in a mesophilic environment. In addition, the growth profiles of the pectinolytica subspecies and Y577 were different, which indicates, in agreement with the overall genomic organization (Fig. 1), that they may belong to different subspecies with a near common ancestor. This is also possible for Y47 and Y567 since they do not present the same growth profile.

Interestingly, the isolate representing the smithia subspecies grew minimally at $18{ }^{\circ} \mathrm{C}$ and not at all at $37{ }^{\circ} \mathrm{C}$ (Fig. 2). According to the Bergey's Manual of Systematic Bacteriology [1], the requirements of this subspecies are not different than those of the other salmonicida subspecies. However, we cannot rule out the possibility that 

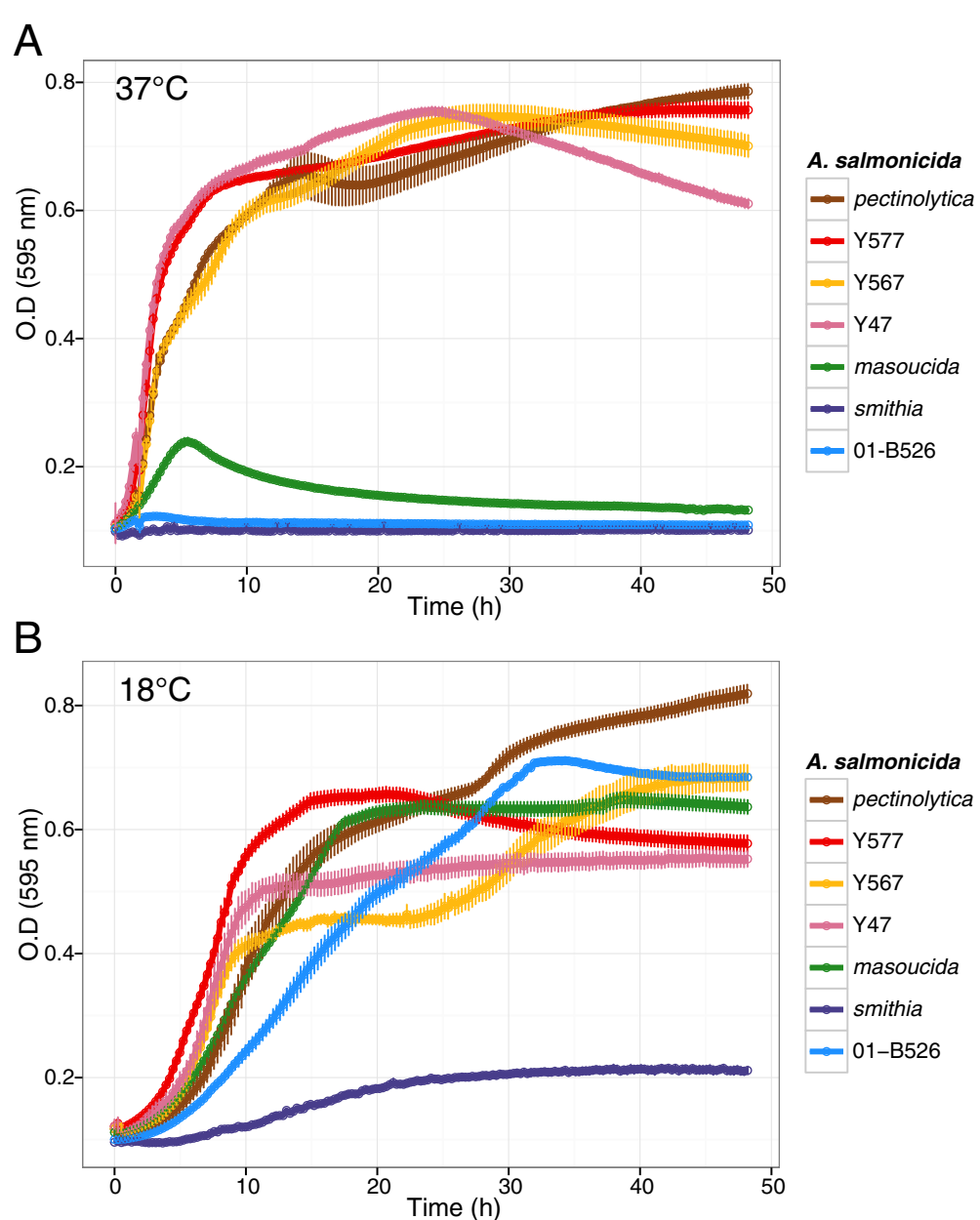

Fig. 2 Growth curves at $37{ }^{\circ} \mathrm{C}(\mathbf{a})$ and $18{ }^{\circ} \mathrm{C}$ (b) for selected A. salmonicida subspecies. The growth curves were determined three times in independent experiments. The means of three replicates with standard error of the means are shown for each subspecies

the smithia subspecies has specific unknown growth requirements.

\section{Genomic features responsible for the dichotomy}

Based on our dataset, it is tempting to suggest that $A$. salmonicida subsp. masoucida lies at the interface of a mesophilic/psychrophilic lifestyle. A. salmonicida could be an interesting model to study how bacteria gradually evolve from a mesophilic to a psychrophilic lifestyle. We postulate here that, from an evolutionary point of view, A. salmonicida is as an example of a recent evolution in lifestyle.

Previous interesting studies have investigated bacteria from different genera to uncover the genomic elements responsible for the mesophilic and psychrophilic lifestyles [31, 32]. However, comparing bacteria from different genera to infer how this type of adaptation has occurred is biased by the noise of unrelated genotypic variations. As reviewed elsewhere [33], bacterial adaptation to different temperatures implies many major physiological changes. Since mesophilic and psychrophilic isolates of $A$. salmonicida are phylogenetically close to each other, genomic variations among these isolates should mostly be related to differences in lifestyle (i.e., mesophilic versus psychrophilic).

Many studies have reported differences in the amino acid compositions of proteins from psychrophilic and mesophilic strains [31, 32, 34, 35]. The ratios of the amino acids of A. salmonicida isolates that can grown at $37^{\circ} \mathrm{C}$ and those that cannot were computed, and the results were analyzed using an unpaired $t$-test (see Additional file 3). Unlike other studies, we found few significant $(p<0.05)$ differences in amino acid composition (only for Gly, His, and Val) between the two groups. This result was expected given that there was no significant difference between the $\% \mathrm{G}+\mathrm{C}$ values of the two groups (see Additional file 3 ). However, it is important to note that we were limited by the small number of salmonicida subspecies, which resulted in a low statistical power (four mesophilic isolates and three psychrophilic isolates). In addition, it has been 
already reported that such analyses may give inconsistent results [36].

The total number of tRNA genes (ttRNA) and tRNA diversity (dtRNA) are also correlated with the optimal growth temperature [37], with the psychrophilic bacteria harboring more tRNA genes than the mesophilic ones in order to compensate for a lower diffusion rate. However, despite the low statistical power no significant difference was found between the A. salmonicida mesophilic and psychrophilic isolates (see Additional file 3). The fact that there was no significant difference between mesophilic and psychrophilic A. salmonicida subspecies in terms of genomic elements that can normally be used to discriminate between lifestyles tends to reinforce the hypothesis that the dichotomy in A. salmonicida lifestyles is a recent event and that one lifestyle could be derived from the other (i.e., some strains have lost the ability to grow at $37^{\circ} \mathrm{C}$ ).

We used an in-house Perl script (see "Methods" section) to find the pan-genome of A. salmonicida in order to shed light on the genomic elements responsible for this dichotomy. The resulting binary matrix (i.e., presence/absence) was used to map the characters (i.e., the genes) on a phylogenetic tree based on the core genome (Fig. 3). This analysis made it possible to determine which genes were acquired and which were lost during evolution and, consequently, may have played a role in the adaption of a given isolate. Given the mesophilic-to- psychrophilic gradient, we investigated the gene repertoires for the branch separating A. salmonicida subsp. masoucida from the mesophilic isolates (branch 1) and the branch separating A. salmonicida subsp. masoucida from the psychrophilic isolates (branch 2) (Fig. 3).

The functional categories of the genes gained and lost at branches 1 and 2 inferred their potential roles (see Additional file 1). The genes related to branch 1 were present in many functional categories, which made sense given that the mesophilic-to-psychrophilic transition is a complex process and cannot be reduced to a few genes or functional categories. However, it is interesting to note that some categories had acquired or lost many genes. The three most affected categories are transcription $(\mathrm{K})$, cell motility $(\mathrm{N})$, and mobilome (X) (Fig. 3). In the case of branch 2, the three functional categories exhibiting most important changes are energy production and conversion (C) (only losses for this category), carbohydrate transport and metabolism (G), replication, recombination, and repair (L) (Fig. 3). Interestingly only gains have been detected for the category related to the mobilome (X) (see Additional file 1).

The $\mathrm{X}$ category (mobilome) appeared to be interesting at both branches. However, since mobile elements are the main cause of contig breaks during the genome assembly process $[16,38]$, the genes in these categories were likely under-estimated by our analysis. As reviewed elsewhere [39], A. salmonicida subsp. salmonicida insertion

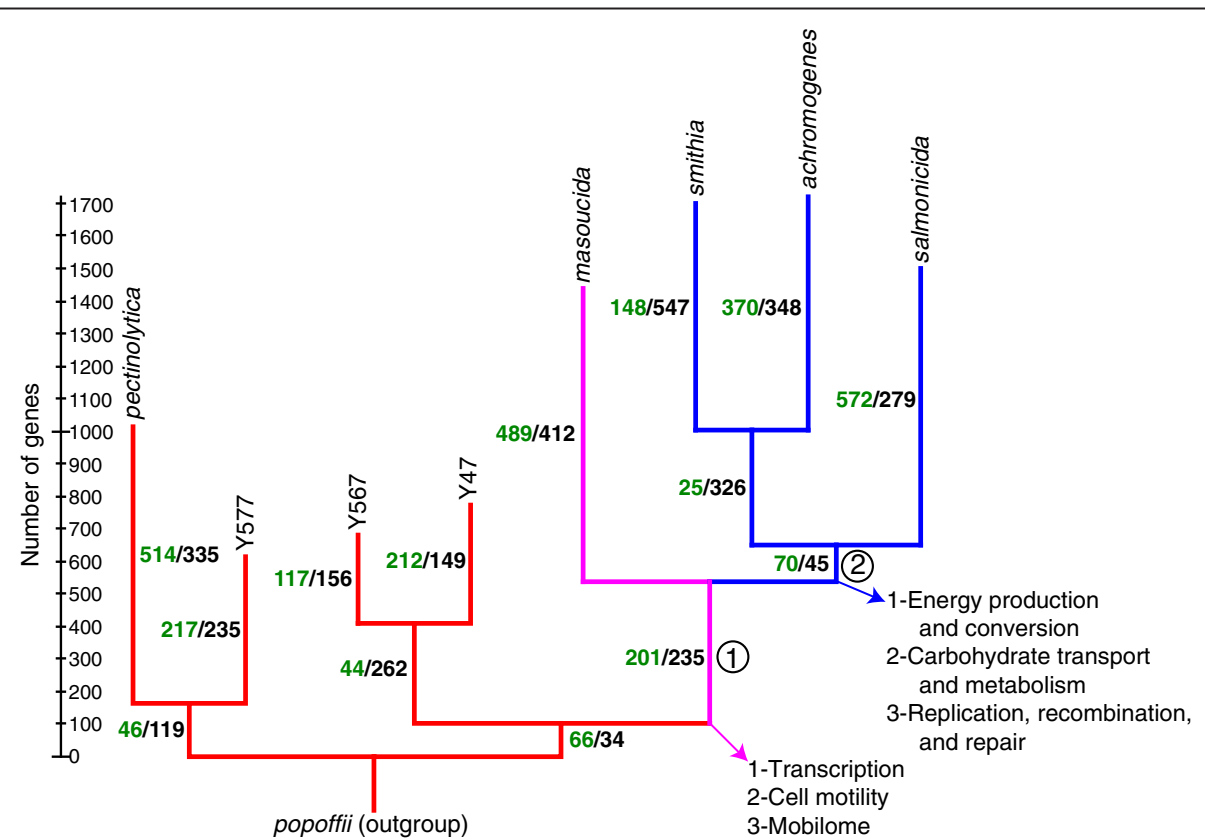

Fig. 3 Distribution of the pan-genome on a phylogenetic tree for some key taxa. The phylogenetic tree was based on the tree found using the core genome. The green and black values indicate the number of genes acquired and lost, respectively, for the specific branch using the parsimonious Dollo model. The branch lengths represent the total number of genes acquired or lost. The three functional categories for which genes are the most affected for branches 1 and 2 are indicated. For more details on the analysis of the functional categories see Additional file 1. For A. salmonicida subsp. salmonicida the strain used was 01-B526 
sequences (ISs) are involved in large-scale mutations such as the loss of the type three secretion system (TTSS) $[15,40]$ (see also Additional file 1), loss of the pAsal1 plasmid [41], the formation of the pAsa6 plasmid from pAsa5 [42], and the disruption of vapA [43], a gene encoding the A-layer [44]. More importantly, ISAS11, which is involved in the loss of the TTSS locus and the pAsal1 plasmid, is more active when the temperature reaches $25{ }^{\circ} \mathrm{C}$ and above [15, 41]. Lastly, the distribution of ISAS4 (also known as IS630) in aeromonads is even enough to construct a clustering of the genus [45], which showed without doubt that salmonicida subspecies isolates harbor large numbers of ISAS4. Given all of the above, especially knowing that the ISAS11 is temperature-sensitive, it is tempting to speculate that ISs are involved in the mesophilic-topsychrophilic transition.

\section{The involvement of ISs in lifestyle evolution}

All the genomes in the present study were in a draft state, with the exception of the A. salmonicida subsp. salmonicida A449 reference strain [9]. Rigorous study of IS diversity in these genomes is complicated since these mobile elements are one of the main factors behind contig breaks during de novo assembly [16, 38, 46]. However, since the drawback is mainly an algorithmic one during the de novo assembly process, we decided to address this issue by working directly with sequencing reads. The high sequencing depth provided by Illumina technology allowed us to study IS diversity directly from the raw sequencing data. As indicated in the "Methods" section, the relative abundance of 70 ISs known to be present in Aeromonas was computed for the studied members of the salmonicida species.

The results of our study of IS diversity showed (1) a dichotomy between mesophilic and the psychrophilic isolates for their IS repertoire and (2) globally a higher number of ISs in the psychrophilic isolates (Fig. 4). In fact, among the 70 ISs studied, 8 were significantly present in the mesophilic isolates and it was possible to see a gradient following the phylogenetic position (Fig. 4a). In the case of the psychrophilic isolates, a striking observation was the high amount of the ISs for the subspecies masoucida and smithia (Fig. 4b). The ISAs4 was the most abundant IS for both masoucida and smithia. This IS was previously postulated to be in high copy number in A. salmonicida subspecies, at exception of the salmonicida subspecies [25]. As indicated previously, ISAS11, which causes major rearrangements at high temperatures, was one of the most common ISs in the psychrophilic isolates. For example, the total assembly of the smithia subspecies was shown to harbor a very high abundance of ISAS11. This observation suggested that the probability of composite transposons is extremely high and, as such, the probability of large-scale genomic rearrangements is also extremely high.

The high copy number of ISAS11 in the smithia subspecies can be partially explained by the presence of the small-high-copy plasmid pJF4097 (see Additional file 1). This plasmid, which is in approximately 40 copies and bears an ISAS11, significantly increased the absolute number of ISAS11 copies. This is a similar situation to that of the salmonicida subspecies like 01-B526 in which the pAsal1 plasmid makes an important contribution to

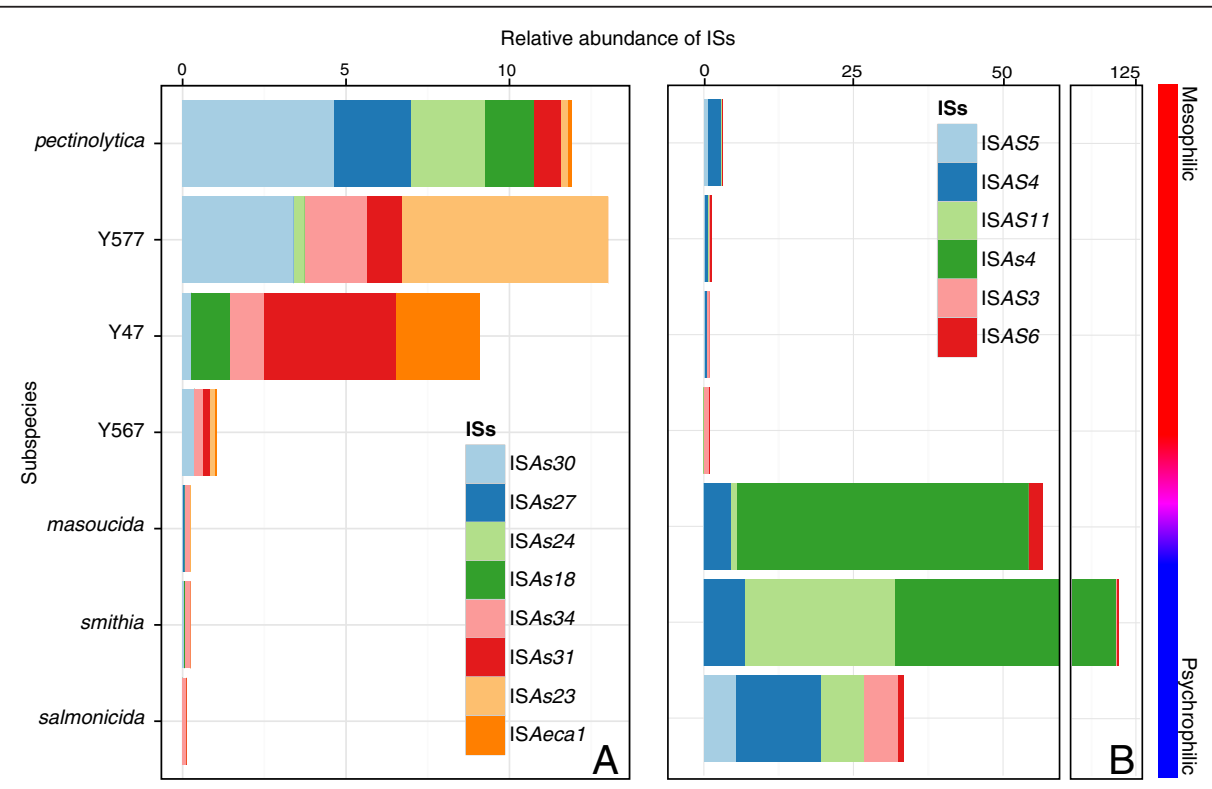

Fig. 4 Relative abundance of ISs in A. salmonicida subspecies. The distribution of 8 ISs significantly found in the mesophilic isolates (a) and 6 in the psychrophilic isolates (b) have been determined in a representative set of sequenced $A$. salmonicida isolates 
the ISAS11 pool [47]. Of note, both plasmids have different replicons, pJF4097 is a ColE1-type whereas pAsal1 is a ColE2-type replicon. The smithia subspecies grew much more slowly at $18{ }^{\circ} \mathrm{C}$ than the other psychrophilic isolates (Fig. 2b). It is possible that the metabolic burden caused by the high-copy plasmid and the ISs are responsible for the slow growth of this isolate at $18{ }^{\circ} \mathrm{C}$. In fact, we found that around $14 \%$ of the subsp. smithia genome was devoted to ISs. As reviewed recently, ISs have a major impact on genome architecture and evolution and an IS expansion might eventually results in a significant reduction of the genome [48]. This genome reduction might significantly change the bacterial lifestyle, for example by enhancing the transition of a free-living to hostdependent bacteria [48]. It is not impossible that the lifestyle of A. salmonicida subsp. smithia is currently experiencing dependence to its host, thus explaining the low growth of the bacterium under laboratory conditions.

We believe that it is important to take the absolute number of ISs into consideration since even plasmidic ISs can undergo rearrangements with genomic ISs. For example, a manual investigation of the draft genome of the A. salmonicida subsp. achromogenes AS03 strain [13] revealed the presence of an ISAS11 adjacent to the aopP gene in a chromosomal contig, just like the pAsal1 plasmid [47].

As recently reviewed [48], ISs not only play significant role in bacterial evolution by shaping the genomic architecture, they can also (1) insert into genes and thus generate pseudogenes and (2) influence expression of neighbor genes. These two genetic impacts are subtler than largescale deletions or rearrangements, but significantly alter the bacterial behavior, regulation, and lifestyle. Consequently we investigated the pseudogenes annotated in the reference strain A449 [9]. On all 155 known pseudogenes, $21(13.5 \%)$ were caused by the insertion of an IS. From these 21 pseudogenes, only one could make sense in a context of psychrophilic/mesophilic lifestyle dichotomy: ASA_1469, which was disrupted by an ISAS4. This gene encodes a dihydrolipoamide acetyltransferase, a protein member of the pyruvate dehydrogenase complex. This complex has been associated to bacterial lifestyle adaptation [49-51]. An investigation of the sequences of every $A$. salmonicida isolate included in this study showed that all the mesophilic subspecies (pectinolytica, Y577, Y567 and Y47) harbor a complete and likely functional gene (i.e. ASA_1469) whereas the psychrophilic subspecies (smithia, achromogenes and salmonicida) and the intermediate one (masoucida) have a truncated gene caused by an insertion of ISAS4 at the same site. This suggests that ISAS4 was inserted in the gene in the common ancestor of all the psychrophilic (including masoucida) subspecies.

Taking altogether, we postulate that ISs play a significant role in the genomic evolution of the salmonicida species and that the psychrophilic subspecies may be "locked" into their lifestyle to conserve their genomic integrity. As shown in other studies and confirmed by the present study (see Additional file 1), ISAS11s induce major genomic instabilities and prevent bacteria harboring them from growing without inducing genomic rearrangements at $25{ }^{\circ} \mathrm{C}$ and above. Moreover, the ISs ISAS1 and ISAS2 are also known to be more active around $30{ }^{\circ} \mathrm{C}$ [43]. Given all of this, it is reasonable to postulate that other ISs may also induce deletions and rearrangements at higher temperature. In fact, although temperaturesensitive ISs were not frequently documented, there was at least one other case in Burkholderia multivorans which was reported [52]. Given the present study, these ISs (i.e., the temperature-sensitive) should be investigated in other psychrophilic bacteria to verify if they can be implied in lifestyle related to growth temperature.

\section{Genes under positive selection for specific lineages}

A. salmonicida can be seen as an ideal model for studying relationships between genomic features and bacterial lifestyles given the spectrum of lifestyles of the various subspecies. It would be interesting to determine whether some genes have undergone a positive selection in specific lineages. The core genome of $A$. salmonicida, composed of a balanced dataset of mesophilic and psychrophilic isolates, contained 2758 genes (see Additional file 4), 322 of which were under positive selection in at least one lineage (see the "Methods" section for the details on how the analyses were performed). The categorization of the genes based on lifestyle revealed that those in mesophilic lineages had undergone a more extensive positive selection process than in the psychrophilic lineages (Fig. 5). This leads to at least two hypotheses, which are not mutually exclusive. The first hypothesis is that the mesophilic isolates are able to grow more efficiently than the psychrophilic isolates (Fig. 2) and consequently replicate their genomes more often, making them more prone to accumulating mutations that allow them to respond more quickly to changes in their environment. The second hypothesis is that, since the mesophilic isolates grow well over a wide range of temperatures, many of their genes are subjected to evolutionary pressure to be able to efficiently colonize and adapt to different environments and hosts (which are actually unknown), unlike the psychrophilic isolates, which are subject to less positive selection. However, this does not explain why the psychrophilic isolates evolved from mesophilic isolates.

\section{Conclusions}

Our dataset, which is based on a robust core genome molecular phylogeny, revealed that $A$. salmonicida isolates are much more diverse than previously thought and that they run the gamut of mesophilic, intermediate, 


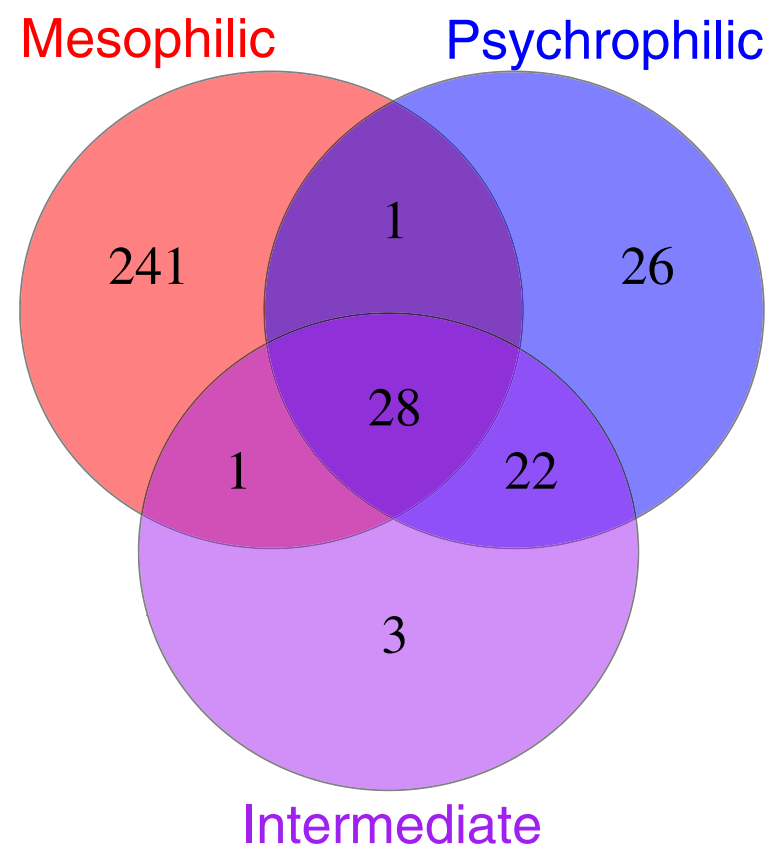

Fig. 5 Venn diagram representing the genes under positive selection in the mesophilic, psychrophilic, and intermediate lineages

and psychrophilic lifestyles. Since mesophilic isolates grow better at $18{ }^{\circ} \mathrm{C}$ than psychrophilic isolates, the psychrophilic lifestyle of $A$. salmonicida isolates may be the result of genetic drift rather than adaptation. Our dataset revealed that the ISs may be an important driving and genomic modeling force that pushed and locked the psychrophilic isolates into this lifestyle, much like the transposable elements that have been shown to drive the evolution of Drosophila melanogaster [53, 54] and ants [55]. On a more fundamental level, our study provided another important proof-of-concept that selfish DNA can produce evolutionary innovations. However, while it is still unclear why psychrophilic isolates have been conserved throughout the evolution of A. salmonicida, there are some possible explanations, including (1) an adaptive selection for psychrophilic hosts like salmonids, (2) a neutral selection, (3) a bottleneck that reduced the effective size of a sub-population of A. salmonicida, allowing an enhanced genetic drift and the fixation of the psychrophilic effect in the population, which seems caused by the invasion of ISs, or (4) a relaxation of the selective pressure to grow in mesophilic environments.

\section{Methods}

\section{Bacterial isolates and growth conditions}

The A. salmonicida subsp. smithia JF4097 isolate was isolated from a diseased Arctic char (Salvelinus alpinus) during an ulcerative and hemorrhagic outbreak in Austria [56]. The A. salmonicida subsp. salmonicida RS
534 isolate, which is also known as A450 [57], was isolated in France. The Indian Y47, Y567, and Y577 isolates were isolated from a chicken and two species of fish (Ompok bimaculatus and Aristichthys nobilis), respectively, sold as food at a local market in Mumbai [58]. The isolates used in our study were grown on a furunculosis agar [59] at $18{ }^{\circ} \mathrm{C}$ for 24 to $72 \mathrm{~h}$. No ethical approval was necessary for any aspect of this study.

\section{DNA extraction, sequencing, and assembling}

The total genomic DNAs of the five isolates were extracted using DNeasy Blood and Tissue kits (Qiagen, Canada). The sequencing libraries were prepared using KAPA Hyper Prep kits and were sequenced by NGS on a MiSeq instrument (Illumina technology) by the Plateforme d'Analyse Génomique of the Institut de Biologie Intégrative et des Systèmes (IBIS, Université Laval). The resulting sequencing reads were assembled de novo into contigs using the A5-miseq pipeline version 20140401 [60]. The assemblies were annotated through the NCBI Prokaryotic Genome Annotation Pipeline (PGAP) and deposited under accession numbers [GenBank:JZTF000 00000, GenBank:JZTG00000000, GenBank:JZTH000000 00, GenBank:JZTI00000000, GenBank:JYFF00000000] for Y47, Y567, Y577, JF4097, and RS 534, respectively. The total genomic DNAs of $A$. salmonicida subsp. pectinolytica $34 \mathrm{mel}^{\mathrm{T}}$ and A. salmonicida subsp. masoucida NBRC $13784^{\mathrm{T}}$ were also extracted and sequenced as described above. However, the DNA of these isolates were only sequenced to compare ISs, as shown in Fig. 4 (see Results and discussion), and were not de novo assembled.

\section{Phylogenetic analyses}

To perform a robust core genome phylogeny, we wrote an in-house Perl script called CoreFinder.pl that relies on BioPerl modules [61] to find the genes involved in the core genome. The script uses coding sequences extracted from a GenBank file and sequentially performs tblastn [62] searches in fasta or multi-fasta (for draft genomes) files. We used A. hydrophila ATCC $7966^{\mathrm{T}}$ [10], which is the $A$. hydrophila type strain, as a reference. The genome of this strain has been well studied and has a high-quality annotation. The others aeromonads used in the present study are listed in the Additional file 1.

The coding sequences (CDSs) found by CoreFinder.pl that were involved in the core genomes of the 43 isolates studied (see Additional file 2) were extracted using another in-house Perl script, which uses tblastn in loop. All the gene sequences were aligned using MUSCLE version 3.5 [63]. The resulting alignments were filtered with BMGE version 1.12 [64] to remove constant characters. Lastly, all individual filtered alignments were concatenated into a matrix. This matrix and all the in-house scripts are available upon request to the corresponding author. 
The phylogenetic analysis was performed by maximumlikelihood using RAxML version 8.1.17 [65] with the GTR $+\Gamma$ model and 1000 rapid bootstraps. The best-fit model GTR $+\Gamma$ was previously chosen by computing the Akaike Information Criterion (AIC) and the Bayesian Information Criterion (BIC) using jModelTest version 2.1.7 [66] (see Additional file 1). The resulting tree was visualized using FigTree version 1.4.2 (http://tree.bio.ed.ac.uk/ software/figtree/). To represent the tree, we used a midpoint rooting, an effective method in which the root is defined by the midpoint between the two most divergent operational taxonomic units (OTUs) [67]. The complete optimization steps are available in Additional file 1.

\section{Assignment of functional categories}

An in-house Perl script was written to perform blastp searches to find correspondences between our protein sequences and the Clusters of Orthologous Groups (COGs) database (2014 update) maintained by NCBI [68] in order to assign a functional category to each of them.

\section{Alignment of the pseudo-chromosomes}

To compare the general structure of the chromosome for all isolates of the salmonicida species used in this study, we generated pseudo-chromosomes by mapping contigs onto the reference strain A449 of the subspecies salmonicida [9], the only chromosome actually fully assembled for the salmonicida species, using CONTIGuator version 2.7.4 [69]. The pseudo-chromosome sequences generated were aligned using Easyfig version 2.1 [70] with default parameters.

\section{Bacterial growth}

A. salmonicida subsp. pectinolytica $\left(34 \mathrm{mel}^{\mathrm{T}}\right), \mathrm{Y} 577$, Y567, Y47, A. salmonicida subsp. smithia (JF4097), A. salmonicida subsp. masoucida (NBRC $13784^{\mathrm{T}}$ ), and $A$. salmonicida subsp. salmonicida (01-B526) were inoculated on furunculosis agar [59] or on tryptic soy agar (TSA) from frozen stocks and were grown at $18{ }^{\circ} \mathrm{C}$ for 24 to $48 \mathrm{~h}$. The isolates were then inoculated in $2 \mathrm{ml}$ of lysogeny broth (LB) and were incubated at $18{ }^{\circ} \mathrm{C}$ overnight. The turbidity was adjusted to an optical density of 0.1 at $595 \mathrm{~nm}\left(\mathrm{OD}_{595}\right)$, and the cultures were incubated at $18{ }^{\circ} \mathrm{C}$ or $37^{\circ} \mathrm{C}$ with shaking at $200 \mathrm{rpm}$ in a Tecan Infinite F200 PRO microplate reader (Tecan, USA). The ODs were read automatically every $15 \mathrm{~min}$ for $48 \mathrm{~h}$. The experiments were performed in triplicate.

\section{Frequency of occurrence of amino acids, $\% G C$, and tRNA composition}

The frequency of occurrence of amino acids and the \%GC were found for each isolate presented in Additional file 3 using respectively pepstats and geecee included in
EMBOSS version 6.6.0.0 [71]. Finally, the tRNA composition (dtRNA and ttRNA) for each isolate was found using tRNA-scan-SE version 1.3.1 [72] by specifying the bacterial search mode. The unpaired t-tests were performed using the statistical framework R [73].

\section{Pan-genome analyses}

An in-house Perl script was used to find the pangenome of the key taxa (available upon request to the corresponding author). Briefly, the translated coding sequences were extracted from the corresponding GenBank files of each taxon. Since all the genomes had been annotated using PGAP at approximately the same time, there was no bias caused by different annotation processes. The A. popoffii genome served as an outgroup. Reciprocal best blast analyses were then performed using blastp. Genes were considered orthologous if their translated sequences shared at least $60 \%$ similarity over at least $85 \%$ of their length. The script encoded the pangenome as a binary matrix (presence/absence). The genes were considered as Dollo characters in MacClade version 4.08 [74] and were mapped on a phylogenetic tree based on the tree generated using the core genome. This approach has been used in other studies $[75,76]$.

\section{Relative IS abundance}

The relative abundances of 70 known ISs for the aeromonads listed in ISfinder [77] were determined using the sequencing reads. Briefly, the sequencing reads for each taxon were filtered using Trimmomatic version 0.32 [78] with the parameters suggested in the manual. The resulting filtered sequencing reads were then mapped using BWA (BWA-MEM algorithm) version 0.7.9a-r786 [79] on contigs sequences resulting from a de novo assembly (see above for the assembly process) to verify the number of high-quality reads. The reads were then aligned on the 70 IS sequences still using BWA (BWA-MEM algorithm) version 0.7.9a-r786 [79]. The total abundance of each IS was determined by comparing the mapped reads over the total number of highquality reads and standardized for the IS length using the results of tools included in SAMtools version 0.1.19$44428 \mathrm{~cd}[80]$.

\section{Genes under selection}

Since we knew the lifestyles and molecular phylogeny of the isolates, we determined whether some genes were under positive selection $(\mathrm{dN} / \mathrm{dS}>1)$ for specific lineages. The core genome was found using the same dataset of eight $A$. salmonicida isolates used to find the pangenome and using the same method used to construct the molecular phylogeny. The well-annotated A. salmonicida subsp. salmonicida A449 chromosome [9] was used as a reference in this case. All the sequences were 
codon-aligned using PRANK version 140603 [81]. All the aligned gene sequences were assessed with HyPhy version 2.2.4 [82] using the adaptive branch-site random effects likelihood (aBSREL) method [83]. A gene was considered under positive selection for the tested lineage if the $p$-value (using the Holm-Bonferroni method in HyPhy) was below 0.05 .

\section{Availability of supporting data}

The sequence datasets obtained during this project have been deposited in the NCBI GenBank database under the accession numbers [GenBank: JZTF00000000, GenBank: JZTG00000000, GenBank: JZTH00000000, GenBank: JZTI00000000, GenBank: JYFF00000000] for Y47, Y567, Y577, JF4097, and RS 534, respectively. The phylogenetic matrix and the in-house Perl scripts are available upon request to the corresponding author.

\section{Additional files}

Additional file 1: Contains additional experimental procedures and results (Table S1. Aeromonads used in the study; Table S2. The five best models and their $-\mathrm{InL}, \mathrm{AIC}$, and BIC values; Table S3. Assembly results; Table S4. Phylogenetic features; Table S5. Biochemical tests used for the mesophilic A. salmonicida strains; Figure S1. Conceptual schematization of the in-house CoreFinder.pl Perl script; Figure S2. Number of genes involved in the core genome based on the similarity percent used with the CoreFinder.pl script; Figure S3. Relative abundance of 26 functional categories for genes used to construct the phylogenetic matrixes at 40 and $80 \%$ similarity; Figure S4. Molecular core genome phylogeny of 43 aeromonads ( $80 \%$ similarity); Figure S5. Molecular phylogeny of 43 aeromonads ( $40 \%$ similarity); Figure $\mathbf{S 6}$. Average nucleotide identity (ANI) analyses for some A. salmonicida subspecies included in this study. Figure S7. Growth curves at $7{ }^{\circ} \mathrm{C}$ for selected $A$. salmonicida subspecies; Figure S8. The three high-copy plasmids found in the Indian strain Y47; Figure S9. The high-copy plasmid pJF4097 found in A. salmonicida subsp. smithia; Figure S10. Result of the PCR assay confirming that the RS 534 strain lost its TTSS by the recombination of two ISAS115; Figure S11. Pan-genome analysis of selected $A$. salmonicida subspecies; Figure S12. Functional categories of the genes under positive selection in the $A$. salmonicida mesophilic lineages). (DOCX $3097 \mathrm{~kb}$ )

Additional file 2: List of the coding sequences (CDSs) being in the core genomes of the 43 aeromonad strains studied. (XLSX $98 \mathrm{~kb}$ )

Additional file 3: The frequency of occurrence of amino acids and the \%GC found for each A. salmonicida isolate. (XLSX $15 \mathrm{~kb}$ )

Additional file 4: List of the coding sequences (CDSs) being in the core genomes of the $8 \mathrm{~A}$. salmonicida strains studied. (XLSX $110 \mathrm{~kb}$ )

\begin{abstract}
Abbreviations
aBSREL: Adaptive branch-site random effects likelihood; ANI: Average nucleotide identities; CDS: Coding sequence; COG: Clusters of orthologous group; IS: Insertion sequence; isDDH: in silico DNA-DNA hybridization; LB: Lysogeny broth; NGS: Next-generation sequencing; OD: Optical density; OTU: Operational taxonomic unit; PGAP: Prokaryotic genome annotation pipeline; RAPD: Random amplified polymorphic DNA; RFLP: Restriction fragment length polymorphism; TSA: Tryptic soy agar; TTSS: Type three secretion system.
\end{abstract}

Competing interests

The authors declare that they have no competing interests.

\section{Authors' contributions}

ATV and SJC. designed the research protocol; ATV, MVT, CGT and VN performed the research; ATV, LF, CGT and SJC. analyzed the data; and ATV, $\mathrm{RCL}$, and SJC. wrote the paper. All authors read and approved the final manuscript.

\section{Acknowledgements}

The authors thank K. H. Tanaka (U. Laval) for her technical support and critical reading of the manuscript, S. Moineau (U. Laval) for his comments and suggestions, J. Frey (U. of Bern) for the JF4097, 34mel ${ }^{\top}$, and NBRC 13784 strains, and R. M. W. Stevenson (U. of Guelph) for the RS 534 strain. ATV received a scholarship from Fonds de recherche du Québec - Nature et technologies (FRQNT). MVT received scholarships from the CREATE program of Ressources Aquatiques Quebec (RAQ). This project was supported by grants from the Natural Sciences and Engineering Research Council of Canada (NSERC), Société de recherche et de développement en aquaculture continentale (SORDAC), RAQ, and the Innovamer program of the Ministère de l'Agriculture, des Pêcheries et de l'Alimentation du Québec. SJC is a research scholar of the Fonds de Recherche du Québec - Santé (FRQS).

\section{Author details}

${ }^{1}$ Institut de biologie intégrative et des systèmes, Pavillon

Charles-Eugène-Marchand, Université Laval, 1030 avenue de la Médecine,

Quebec City G1V OA6QC, Canada. ${ }^{2}$ Centre de recherche de I'Institut universitaire de cardiologie et de pneumologie de Québec (Hôpital Laval), 2725 Chemin Sainte-Foy, Quebec City G1V 4G5QC, Canada. ${ }^{3}$ Département de biochimie, de microbiologie et de bio-informatique, Faculté des sciences et de génie, Université Laval, 1045 avenue de la Médecine, Quebec City G1V OA6QC, Canada. ${ }^{4}$ Département de microbiologie-infectiologie et immunologie, Faculté de médecine, Université Laval, Quebec City, QC, Canada. ${ }^{5}$ Food Technology Division, Bhabha Atomic Research Centre, Mumbai 400085, India.

Received: 9 October 2015 Accepted: 6 January 2016

Published online: 12 January 2016

\section{References}

1. Martin-Carnahan A, Joseph S. Aeromonadales ord. nov. In: Brenner D, Krieg N, Staley J, Garrity G, Boone D, De Vos P, editors. Bergey's Manual ${ }^{\oplus}$ Syst. Bacteriol. SE - 12. Springer US. 2005. p. 556-87.

2. Colston SM, Fullmer MS, Beka L, Lamy B, Gogarten JP. Bioinformatic Genome Comparisons for Taxonomic and Phylogenetic Assignments Using Aeromonas as a Test Case. MBio. 2014:5:1-13.

3. Percival SL, Williams DW. Chapter Three - Aeromonas. In: Gray of WD (Second E, editor. London: Academic; 2014. p. 49-64.

4. Palumbo SA, Morgan DR, Buchanan RL. Influence of Temperature, NaCl, and pH on the Growth of Aeromonas Hydrophila. J Food Sci. 1985:50:1417-21.

5. Park S, Ha S-D. Effect of temperature on the growth kinetics and predictive growth model of Aeromonas hydrophila on squid (Sepioteuthis sepioidea). Food Sci Biotechnol. 2014;23:307-12.

6. Pavan ME, Abbott SL, Zorzópulos J, Janda JM. Aeromonas salmonicida subsp. pectinolytica subsp. nov., a new pectinase- positive subspecies isolated from a heavily polluted river. Int J Syst Evol Microbiol. 2000;50:1119-24.

7. Janda JM, Abbott SL. The Genus Aeromonas: Taxonomy, Pathogenicity, and Infection. Clin Microbiol Rev. 2010;23:35-73.

8. Dallaire-Dufresne S, Tanaka KH, Trudel MV, Lafaille A, Charette SJ. Virulence, genomic features, and plasticity of Aeromonas salmonicida subsp. salmonicida, the causative agent of fish furunculosis. Vet Microbiol. 2014;169:1-7.

9. Reith ME, Singh RK, Curtis B, Boyd JM, Bouevitch A, Kimball J, et al. The genome of Aeromonas salmonicida subsp. salmonicida A449: insights into the evolution of a fish pathogen. BMC Genomics. 2008;9:427.

10. Seshadri R, Joseph SW, Chopra AK, Sha J, Shaw J, Graf J, et al. Genome sequence of Aeromonas hydrophila ATCC 7966 T: Jack of all trades. J Bacteriol. 2006;188:8272-82.

11. Nagar V, Shashidhar R, Bandekar JR. Characterization of Aeromonas strains isolated from Indian foods using rpoD gene sequencing and whole cell protein analysis. World J Microbiol Biotechnol. 2013;29:745-52.

12. Valdes N, Espinoza C, Sanhueza L, Gonzalez A, Corsini G, Tello M. Draft Genome Sequence of the Chilean isolate Aeromonas salmonicida strain CBA100. FEMS Microbiol Lett. 2015;362:fnu062. 
13. Han JE, Kim JH, Shin SP, Jun JW, Chai JY, Park SC. Draft Genome Sequence of Aeromonas salmonicida subsp. achromogenes AS03, an Atypical Strain Isolated from Crucian Carp (Carassius carassius) in the Republic of Korea. Genome Announc. 2013;1:e00791-13.

14. Roger F, Marchandin H, Jumas-Bilak E, Kodjo A, Lamy B. Multilocus genetics to reconstruct aeromonad evolution. BMC Microbiol. 2012;12:62.

15. Emond-Rheault J-G, Vincent AT, Trudel MV, Frey J, Frenette M, Charette SJ. AsaGEl2b: a new variant of a genomic island identified in the Aeromonas salmonicida subsp. salmonicida JF3224 strain isolated from a wild fish in Switzerland. FEMS Microbiol Lett. 2015;362:fnv093.

16. Vincent AT, Tanaka KH, Trudel MV, Frenette M, Derome N, Charette SJ. Draft genome sequences of two Aeromonas salmonicida subsp. salmonicida isolates harboring plasmids conferring antibiotic resistance. FEMS Microbiol Lett. 2015;362:1-4.

17. Charette SJ, Brochu F, Boyle B, Filion G, Tanaka KH, Derome N. Draft genome sequence of the virulent strain 01-B526 of the fish pathogen Aeromonas salmonicida. J Bacteriol. 2012;194:722-3.

18. Pavan ME, Pavan EE, López NI, Levin L, Pettinari MJ. Living in an extremely polluted environment: clues from the genome of melanin-producing Aeromonas salmonicida subsp. pectinolytica $34 \mathrm{mel}^{\top}$. Appl Environ Microbiol. 2015;81:5235-48

19. Belland RJ, Trust TJ. DNA:DNA reassociation analysis of Aeromonas salmonicida. J Gen Microbiol. 1988;134:307-15.

20. García JA, Larsen JL, Dalsgaard I, Pedersen K. Pulsed-field gel electrophoresis analysis of Aeromonas salmonicida ssp. salmonicida. FEMS Microbiol Lett. 2000;190:163-6.

21. Hänninen ML, Ridell J, Hirvelä-Koski V. Phenotypic and molecular characteristics of Aeromonas salmonicida subsp. salmonicida isolated in southern and northern Finland. J Appl Bacteriol. 1995;79:12-21.

22. O'hlci B, Olivier G, Powell R. Genetic diversity of the fish pathogen Aeromonas salmonicida demonstrated by random amplified polymorphic DNA and pulsed-field gel electrophoresis analyses. Dis Aquat Organ. 2000;39:109-19.

23. Umelo E, Trust TJ. Physical map of the chromosome of Aeromonas salmonicida and genomic comparisons between Aeromonas strains. Microbiology. 1998;144:2141-9.

24. McCormick WA, Stevenson RM, Maclnnes Jl. Restriction endonuclease fingerprinting analysis of Canadian isolates of Aeromonas salmonicida. Can J Microbiol. 1990;36:24-32.

25. Nilsson WB, Gudkovs N, Strom MS. Atypical strains of Aeromonas salmonicida contain multiple copies of insertion element ISAsa4 useful as a genetic marker and a target for PCR assay. Dis Aquat Organ. 2006;70:209-17.

26. Emond-Rheault J-G, Vincent AT, Trudel MV, Brochu F, Tanaka KH, Attéré SA, et al. Variants of a genomic island in Aeromonas salmonicida subsp. salmonicida link isolates with their geographical origins. Vet. Microbiol. 2015;175:68-76.

27. Austin DA, McIntosh D, Austin B. Taxonomy of Fish Associated Aeromonas spp., with the Description of Aeromonas salmonicida subsp. smithia subsp. nov. Syst Appl Microbiol. 1989;11:277-90.

28. Beaz-Hidalgo R, Figueras MJ. Molecular Detection and Characterization of Furunculosis and Other Aeromonas Fish Infections. In: Carvalho E, editor. Heal. Environ. Aquac. Intech. 2012. p. 97-132.

29. Dautremepuits C, Fortier M, Croisetiere S, Belhumeur P, Fournier M. Modulation of juvenile brook trout (Salvelinus fontinalis) cellular immune system after Aeromonas salmonicida challenge. Vet Immunol Immunopathol. 2006;110:27-36

30. Dacanay A, Knickle L, Solanky KS, Boyd JM, Walter JA, Brown LL, et al. Contribution of the type III secretion system (TTSS) to virulence of Aeromonas salmonicida subsp. salmonicida. Microbiology. 2006;152:1847-56.

31. Metpally RPR, Reddy BVB. Comparative proteome analysis of psychrophilic versus mesophilic bacterial species: Insights into the molecular basis of cold adaptation of proteins. BMC Genomics. 2009;10:11.

32. Methé BA, Nelson KE, Deming JW, Momen B, Melamud E, Zhang X, et al. The psychrophilic lifestyle as revealed by the genome sequence of Colwellia psychrerythraea $34 \mathrm{H}$ through genomic and proteomic analyses. Proc Natl Acad Sci U S A. 2005:102:10913-8.

33. Barria C, Malecki M, Arraiano CM. Bacterial adaptation to cold. Microbiol (United Kingdom). 2013;159:2437-43.

34. Riley M, Staley JT, Danchin A, Wang TZ, Brettin TS, Hauser LJ, et al. Genomics of an extreme psychrophile, Psychromonas ingrahamii. BMC Genomics. 2008:9:210.

35. Saunders NFW, Thomas T, Curmi PMG, Mattick JS, Kuczek E, Slade R, et al. Mechanisms of thermal adaptation revealed from the genomes of the
Antarctic Archaea Methanogenium frigidum and Methanococcoides burtonii. Genome Res. 2003:13:1580-8.

36. D'Amico S, Collins T, Marx J-C, Feller G, Gerday C. Psychrophilic microorganisms: challenges for life. EMBO Rep. 2006;7:385-9.

37. Satapathy SS, Dutta M, Ray SK. Higher tRNA diversity in thermophilic bacteria: A possible adaptation to growth at high temperature. Microbiol Res. 2010;165:609-16.

38. Vincent AT, Boyle B, Derome N, Charette SJ. Improvement in the DNA sequencing of genomes bearing long repeated elements. J Microbiol Methods. 2014;107:186-8.

39. Tanaka KH, Frenette M, Charette SJ. IS-mediated loss of virulence by Aeromonas salmonicida: A tangible piece of an evolutionary puzzle. Mob Genet Elements. 2013:3:e23498.

40. Daher RK, Filion G, Tan SGE, Dallaire-Dufresne S, Paquet VE, Charette SJ. Alteration of virulence factors and rearrangement of pAsa5 plasmid caused by the growth of Aeromonas salmonicida in stressful conditions. Vet Microbiol. 2011;152:353-60.

41. Tanaka KH, Dallaire-Dufresne S, Daher RK, Frenette M, Charette SJ. An Insertion Sequence-Dependent Plasmid Rearrangement in Aeromonas salmonicida Causes the Loss of the Type Three Secretion System. PLoS One. 2012; 7:e33725.

42. Najimi M, Balado M, Lemos ML, Osorio CR. Genetic characterization of pAsa6, a new plasmid from Aeromonas salmonicida subsp. salmonicida that encodes a type III effector protein AopH homolog. Plasmid. 2009;61:176-81.

43. Gustafson CE, Chu S, Trust TJ. Mutagenesis of the paracrystalline surface protein array of Aeromonas salmonicida by endogenous insertion elements. J Mol Biol. 1994;237:452-63.

44. Chu S, Cavaignac S, Feutrier J, Phipps BM, Kostrzynska M, Kay WW, et al. Structure of the tetragonal surface virulence array protein and gene of Aeromonas salmonicida. J Biol Chem. 1991;266:15258-65.

45. Studer N, Frey J, Vanden BP. Clustering subspecies of Aeromonas salmonicida using IS630 typing. BMC Microbiol. 2013;13:13-36.

46. Beatson SA, Walker MJ. Tracking antibiotic resistance. Science. 2014:345:1454-5.

47. Fehr D, Casanova C, Liverman A, Blazkova H, Orth K, Dobbelaere D, et al. AopP, a type III effector protein of Aeromonas salmonicida, inhibits the NFkappaB signalling pathway. Microbiology. 2006;152:2809-18.

48. Siguier $\mathrm{P}$, Gourbeyre $\mathrm{E}_{1}$ Chandler M. Bacterial insertion sequences: Their genomic impact and diversity. FEMS Microbiol Rev. 2014;38:865-91.

49. De Kok A, Hengeveld AF, Martin A, Westphal AH. The pyruvate dehydrogenase multi-enzyme complex from Gram-negative bacteria. Biochim Biophys Acta. 1998;1385:353-66.

50. Dziewit L, Bartosik D. Plasmids of psychrophilic and psychrotolerant bacteria and their role in adaptation to cold environments. Front Microbiol. 2014:5:596.

51. Qiu Y, Kathariou S, Lubman DM. Proteomic analysis of cold adaptation in a Siberian permafrost bacterium-Exiguobacterium sibiricum 255-15 by twodimensional liquid separation coupled with mass spectrometry. Proteomics. 2006;6:5221-33

52. Ohtsubo Y, Genka H, Komatsu H, Nagata Y, Tsuda M. High-temperatureinduced transposition of insertion elements in Burkholderia multivorans ATCC 17616. Appl Environ Microbiol. 2005;71:1822-8.

53. González J, Lenkov K, Lipatov M, Macpherson JM, Petrov DA. High rate of recent transposable element-induced adaptation in Drosophila melanogaster. PLoS Biol. 2008;6:2109-29.

54. González J, Petrov DA. The adaptive role of transposable elements in the Drosophila genome. Gene. 2009;448:124-33.

55. Schrader L, Kim JW, Ence D, Zimin A, Klein A, Wyschetzki K, et al. Transposable element islands facilitate adaptation to novel environments in an invasive species. Nat Commun. 2014;5:5495.

56. Goldschmidt-Clermont E, Hochwartner O, Demarta A, AP C, Frey J. Outbreaks of an ulcerative and haemorrhagic disease in Arctic char Salvelinus alpinus caused by Aeromonas salmonicida subsp. smithia. Dis Aquat Organ. 2009:86:81-6.

57. Belland RJ, Trust TJ. Cloning of the gene for the surface array protein of Aeromonas salmonicida and evidence linking loss of expression with genetic deletion. J Bacteriol. 1987;169:4086-91.

58. Nagar V, Shashidhar R, Bandekar JR. Prevalence, characterization, and antimicrobial resistance of Aeromonas strains from various retail food products in Mumbai. India J Food Sci. 2011;76:M486-92.

59. Hänninen ML, Hirvelä-Koski V. Molecular and phenotypic methods for the characterization of atypical Aeromonas salmonicida. Vet Microbiol. 1997;56:147-58. 
60. Coil D, Jospin G, Darling AE. A5-miseq : an updated pipeline to assemble microbial genomes from Illumina MiSeq data. Bioinformatics. 2014;31(4):587-9.

61. Stajich JE, Block D, Boulez K, Brenner SE, Chervitz SA, Dagdigian C, et al. The Bioperl toolkit: Perl modules for the life sciences. Genome Res. 2002;12:1611-8.

62. Altschul SF, Madden TL, Schäffer AA, Zhang J, Zhang Z, Miller W, et al. Gapped BLAST and PSI-BLAST: a new generation of protein database search programs. Nucleic Acids Res. 1997;25:3389-402.

63. Edgar RC. MUSCLE: Multiple sequence alignment with high accuracy and high throughput. Nucleic Acids Res. 2004;32:1792-7.

64. Criscuolo A, Gribaldo S. BMGE (Block Mapping and Gathering with Entropy): a new software for selection of phylogenetic informative regions from multiple sequence alignments. BMC Evol Biol. 2010;10:210.

65. Stamatakis A. RAxML version 8: A tool for phylogenetic analysis and postanalysis of large phylogenies. Bioinformatics. 2014;30:1312-3.

66. Darriba D, Taboada GL, Doallo R, Posada D. jModelTest 2: more models, new heuristics and parallel computing. Nat Methods. 2012;9:772.

67. Hess PN, De Moraes Russo CA. An empirical test of the midpoint rooting method. Biol J Linn Soc. 2007;92:669-74.

68. Tatusov RL, Fedorova ND, Jackson JD, Jacobs AR, Kiryutin B, Koonin EV, et al. The COG database: an updated version includes eukaryotes. BMC Bioinformatics. 2003;4:41.

69. Galardini M, Biondi EG, Bazzicalupo M, Mengoni A. CONTIGuator: a bacterial genomes finishing tool for structural insights on draft genomes. Source Code Biol Med. 2011;6:11.

70. Sullivan MJ, Petty NK, Beatson SA. Easyfig: a genome comparison visualiser. Bioinformatics. 2011;27:1009-10.

71. Rice P, Longden I, Bleasby A. EMBOSS: The European Molecular Biology Open Software Suite. Trends Genet. 2000;16:276-7.

72. Lowe TM, Eddy SR. tRNAscan-SE: A program for improved detection of transfer RNA genes in genomic sequence. Nucleic Acids Res. 1997;25:955-64.

73. Team RC. R: A Language and Environment for Statistical Computing. Vienna, Austria: R Found. Stat. Comput; 2013.

74. Maddison WP, Maddison DR. MacClade 4: analysis of phylogeny and character evolution. Version 4.0. Massachusetts: Sinauer Sunderl; 2000.

75. Brouard JS, Otis C, Lemieux C, Turmel M. The exceptionally large chloroplast genome of the green alga Floydiella terrestris illuminates the evolutionary history of the Chlorophyceae. Genome Biol Evol. 2010;2:240-56.

76. Turmel M, Otis C, Lemieux C. Dynamic Evolution of the Chloroplast Genome in the Green Algal Classes Pedinophyceae and Trebouxiophyceae. Genome Biol Evol. 2015;7:2062-82

77. Siguier P, Perochon J, Lestrade L, Mahillon J, Chandler M. ISfinder: the reference centre for bacterial insertion sequences. Nucleic Acids Res. 2006;34:D32-6.

78. Bolger AM, Lohse M, Usadel B. Trimmomatic: a flexible trimmer for Illumina sequence data. Bioinformatics. 2014;30:2114-20.

79. Li H, Durbin R. Fast and accurate short read alignment with BurrowsWheeler transform. Bioinformatics. 2009;25:1754-60.

80. Li H, Handsaker B, Wysoker A, Fennell T, Ruan J, Homer N, et al. The Sequence Alignment/Map format and SAMtools. Bioinformatics. 2009;25:2078-9.

81. Löytynoja A, Goldman N. An algorithm for progressive multiple alignment of sequences with insertions. Proc Natl Acad Sci U S A. 2005:102:10557-62.

82. Kosakovsky Pond SL, Frost SDW, Muse SV. HyPhy: Hypothesis testing using phylogenies. Bioinformatics. 2005;21:676-9.

83. Smith MD, Wertheim JO, Weaver S, Murrell B, Scheffler K, Kosakovsky Pond SL. Less Is More: An Adaptive Branch-Site Random Effects Model for Efficient Detection of Episodic Diversifying Selection. Mol Biol Evol. 2015;32:1342-53.

\section{Submit your next manuscript to BioMed Central and we will help you at every step:}

- We accept pre-submission inquiries

- Our selector tool helps you to find the most relevant journal

- We provide round the clock customer support

- Convenient online submission

- Thorough peer review

- Inclusion in PubMed and all major indexing services

- Maximum visibility for your research

Submit your manuscript at www.biomedcentral.com/submit 